\title{
Erratum to: Bridging the genotyping gap: using genotyping by sequencing (GBS) to add high-density SNP markers and new value to traditional bi-parental mapping and breeding populations
}

\author{
Jennifer Spindel ${ }^{1} \cdot$ Mark Wright $^{1} \cdot$ Charles Chen $^{1}$. \\ Joshua Cobb ${ }^{1} \cdot$ Joseph Gage $^{1} \cdot$ Sandra Harrington ${ }^{1}$. \\ Mathias Lorieux $^{2,3} \cdot$ Nourollah Ahmadi $^{4} \cdot$ Susan McCouch $^{1}$
}

Published online: 31 October 2015

(c) Springer-Verlag Berlin Heidelberg 2015

\section{Erratum to: Theor Appl Genet (2013) 126:2699-2716 DOI 10.1007/s00122-013-2166-x}

Unfortunately, the original publication of this paper contained error in the presentation of Fig. 5.

The ratio of genetic position to physical position (shown on the y-axis of Fig. 5) was incorrectly plotted as cumulative $\mathrm{cM}$ to cumulative $\mathrm{Mb}$. As a result, this figure does not show an estimate of local recombination, as the authors intended, but rather the averaging of genetic recombination along the length of the chromosome, i.e., at the beginning of the chromosome, no averaging takes place, but by the end of the $\mathrm{x}$-axis, the recombination rate has converged to the genome-wide average.

The online version of the original article can be found under doi:10.1007/s00122-013-2166-x.

Susan McCouch

srm4@cornell.edu

Jennifer Spindel

jes462@cornell.edu

Mark Wright

mhw6@ cornell.edu

1 Department of Plant Breeding and Genetics, Cornell University, 162 Emerson Hall, Ithaca, NY 14853-1901, USA

2 UMR DIADE, Institut de Recherche pour le Développement (IRD), 34394 Montpellier Cedex 5, France

3 Rice Genetics and Genomics Laboratory, International Center for Tropical Agriculture (CIAT), AA6713 Cali, Colombia

4 Centre de Coopération Internationale en Recherche Agronomique pour le Développement (CIRAD), TA06/01 Avenue Agropolis, 34398 Montpellier Cedex 05, France
To obtain an estimate of local recombination rate, a Gaussian kernel (Python3 scipy.ndimage.filters.gaussianfilter) was used to obtain arrays of smoothed genetic distance and smoothed physical distance for the 30,984 post-imputation, post-error correction GBS SNPs-note that the SSR data were insufficiently dense to map local recombination, so corrected Fig. 5 shows results using the GBS SNPs only. Three levels of sigma $(\sigma=50,100$, and 200), roughly equivalent to window-size/two in a 'rolling windows' algorithm, were used. Smaller values of sigma allow the visualization of greater detail but also include more noise, while larger values result in greater smoothing and less noisy models. The smoothed genetic position was then divided by the smoothed physical position and plotted versus physical position using pylab (Python3 matplotlib. pyplot).

In the corrected Fig. 5, the authors highlight that recombination rates ranged substantially from as high as four times the genome-wide average to rates half the genome average, excluding centromeric regions. Thus the results still supports the original conclusion that there is benefit to saturating a genetic map of a biparental cross with GBS SNPs as variation in local recombination means that placing one marker per $\mathrm{cM}$ will not ensure genetic linkage between every QTL and at least one marker. The authors also note that in the corrected figure, recombination is suppressed around the centromeres, as is typically expected. 
Fig. 5 Variation in recombination frequency across the genome in the IR64 $\times$ Azucena RIL population, calculated across the genome using final PANATI post-PLUMAGE dataset. For SNPs on each chromosome, the genetic distance and physical distances were smoothed using a Gaussian kernel at three levels of sigma: $\sigma=50$ (green line), $\sigma=100$ (red line), and $\sigma=200$ (blue line). Smoothed genetic distance was then divided by smoothed physical distance and plotted by physical distance. Smaller values of sigma allow the greater visualization of local recombination, but also resulted in noisier models. $1 \mathrm{cM}$ in rice $=240 \mathrm{~Kb}$, so average recombination was expected to be at cM:Mb ratio of 4 , black horizontal line. Peaks above the black line are regions of heightened recombination while valleys below the black line are regions where recombination is suppressed. Pericentromeric regions are delimited by vertical dashed black lines. On all chromosomes, recombination was suppressed around the centromeres, as expected (color figure online)
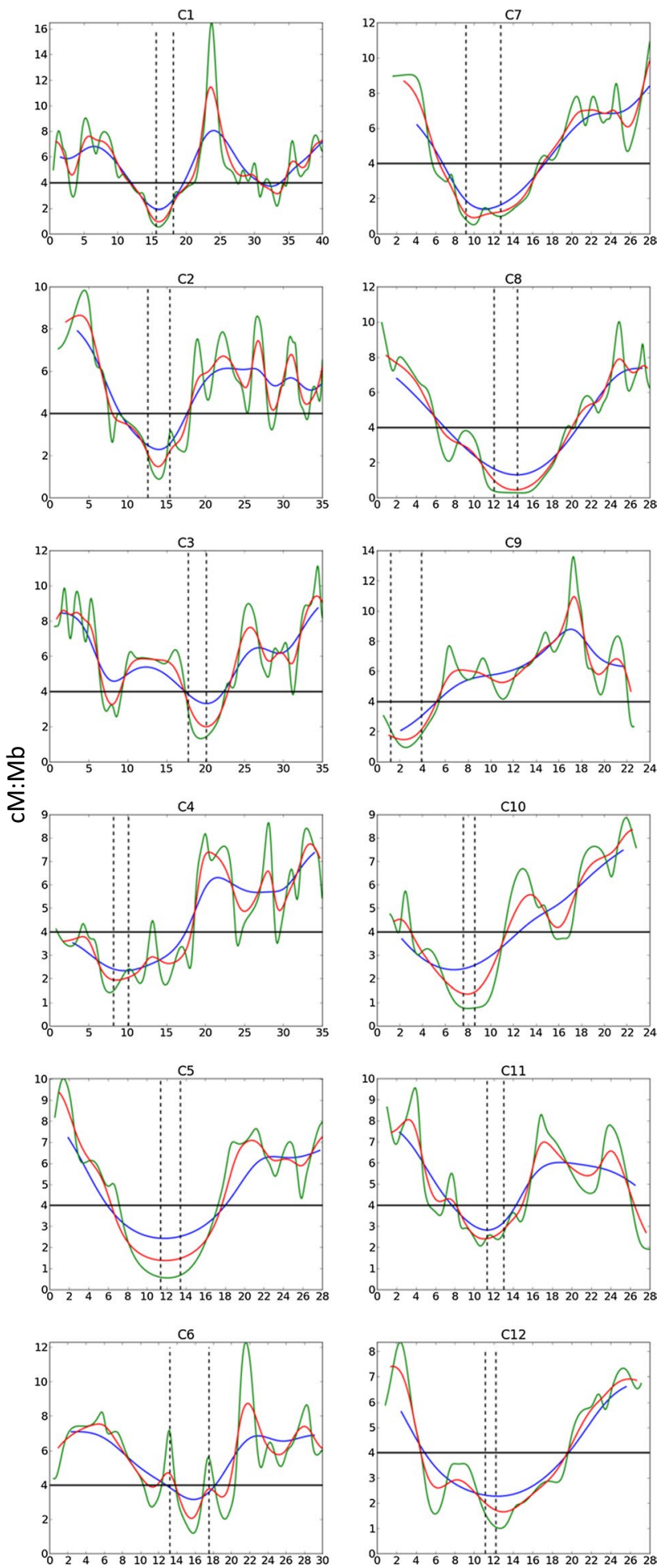

Pos $(\mathrm{Mb})$ 